\title{
Submission Domain Abbreviation
}

National Cancer Institute

\section{Source}

National Cancer Institute. Submission Domain Abbreviation. NCI Thesaurus. Code C49558.

A unique, 2-character domain code used in the regulatory submission process. The domain abbreviation is used consistently throughout the submission, i.e. in the dataset name, as the value of the domain variable within the dataset, and as a prefix for most variable names in the dataset. (CDISC Glossary) 